\title{
A Case Report of Incomplete Carney Complex With SMARCA4-Deficient Thoracic Sarcoma: Implications for the SLC7Aaa and ARID1A Expression
}

yusuke kito ( $\square$ yskkito@gmail.com )

Gifu University Graduate School of Medicine https://orcid.org/0000-0003-1373-986X

Keisuke Kawashima

Gifu University Graduate School of Medicine

Chiemi Saigo

Gifu University Graduate School of Medicine

Masayoshi Hasegawa

Gifu University Graduate School of Medicine

Shusuke Nomura

Gifu University Graduate School of Medicine

Takuya Mikamo

Gifu University Graduate School of Medicine

Yuki Hanamatsu

Gifu University Graduate School of Medicine

Yasuhiro Matsuo

Matsunami General Hospital

Tamostu Takeuchi

Gifu University Graduate School of Medicine

\section{Case Report}

Keywords: SMARCA4-deficient thoracic sarcoma, ARID1A, SLC7A11, Ganglioneuroma, Enchondroma, Incomplete Carney complex

Posted Date: May 24th, 2021

DOl: https://doi.org/10.21203/rs.3.rs-540037/v1

License: (c) (1) This work is licensed under a Creative Commons Attribution 4.0 International License.

Read Full License 


\section{Abstract}

Background: SWI/SNF-related, matrix-associated, matrix-associated, actin-dependent regulator of chromatin, subfamily A, member (SMARCA4)-deficient thoracic sarcoma (SMARCA4-DTS) is a rare disease that has recently been described as an entity. It is characterized by an aggressive clinical course and specific genetic alterations. As an immunohistological feature, the tumors are deficient in SMARCA4 and SMARCA2 and express the sex-determining region Y-box 2 (SOX2). In contrast, Carney's triad is a syndrome that combines three rare soft tissue tumors: gastric leiomyosarcoma, pulmonary chondroma, and extra-adrenal paraganglioma, of which at least two are required for diagnosis. Both diseases are valuable case, and there have been no previous reports of their coexistence.

Case presentation: A 43-year-old man visited our hospital because of respiratory distress. Computed tomography revealed a large mass measuring $55 \mathrm{~mm}$ in the upper lobe of his right lung and front mediastinum, with metastases in the surrounding lymph nodes. Needle biopsy was performed for diagnosis, and histological examination of the samples revealed monotonous epithelioid-like cells with loose binding and sheet-form proliferation. The tumor cells had distinct nuclei, with rhabdoid-kile cells in some locations. Immunohistochemical analysis revealed that the tumor cells were positive for SOX2, CD34, and p53 and negative for SMARCA4 and SMARCA2. The patient died 6 months after admission without any treatment. Autopsy revealed ganglioneuroma and enchondroma, suggesting an incomplete Carney complex.

Conclusion: SMARCA4-DTS is a rare and recently established disease. While it is difficult to siagnose, it is necessary to distinguish undifferentiated carcinoma, large cell carcinoma, Ewing sarcoma, epithelioid sarcoma, etc. when diagnosing tumors involving the mediastinum, In addition, case with both an incomplete Carney complex and SMARCA4-DTS are very rare. We discuss and report about SMARCA4DTS by examining the expression of AT-rich interactive domain-containing protein $1 \mathrm{~A}$ and solute carrier family 7 member 11 .

\section{Background}

SWI/SNF-related, matrix-associated, actin-dependent regulator of chromatin, subfamily A, member 4 (SMARCA4)-deficient thoracic sarcoma (SMARCA4-DTS) is a new disease concept with characteristic clinical, pathological, and molecular features [1]. This tumor is caused by an abnormality in SMARCA4/BRG1, which is a chromatin remodeling mechanism [2-4]. This tumor has been reported in various organs, including small cell carcinoma of ovary, medulloblastoma, lung cancer, intrathoracic sarcoma, and paranasal sinuses [5]. Its clinical feature is its average age at onset, i.e., 40S to 50S, most often in men and smokers. SMARCA4-DTS is refractory to treatment, progresses rapidly, and has a poor prognosis. Its morphological features include monotonous epithelioid-like cells that proliferate in sheet form with loose connectivity [6]. The tumor cells have a large eosinophilic cytoplasm, appear as rhabdoid cells, and have large swollen nuclei and distinct nucleoli [7]. Among the immunohistological features are high positivity for SRY (sex-determining region Y)-box 2 (SOX2), Cd34, Sal-loke protein 4, and p53; keratin 
positive in half of the reported cases; and general negativity for BRG1/SMARCA4, Claudin4, transcription termination factor 1 , desmin, and S100 calcium-binding protein $\mathrm{P}$ (S100P) [8].

In contrast, in 1977, Carney et al. reported a case in which two or more of the three lesions of gastric leiomyosarcoma, extra-adrenal ganglionoma, and pulmonary chondroma were observed as single disease group, irrespective of it being metachronous or simultaneous [9]. In 1977, Carney also reported the clinical features of 79 case of this syndrome with two or more signs as it may take years for all three signs to appear [10]. These cases are considered to correspond to the incomplete form of Carney's triad.

\section{Case Presentation}

A 43-year-old man visited our hospital because of respiratory distress. He was a heavy smoker. Computed tomography revealed large mass measuring 55 in the upper lobe of the right lung and front mediastinum, with multiple metastases in the surrounding lymph nodes. Lymph node biopsy was performed. The initial pathological diagnosis was non-small cell lung cancer and poorly differentiated adenocarcinoma.

Chemotherapy was administered. However, the tumor was refractory, and the patient died 6 months after admission. Subsequently, an autopsy was performed. Macroscopically, the tumor was $10 \mathrm{~cm}$ in size from the upper lobe of the right lung to the anterior mediastinum, with many lymph node metastases observed, the tumor was deeper than the submucosa and $10 \mathrm{~cm}$ in size, solid, opalescent, and necrotic.

Histologically, the tumor showed a sheet-like growth pattern of monotonous epithelial-like cells. The tumor cells showed loose binding and distinct nucleoli, with rhabdoid-like cells observed in some places. Frequent mitotic figures and necrosis were observed. Representative microscopic morphological features are shown in Fig. 1A and 1B. Subsequently, immunohistochemical staining was performed on paraffinembedded tissue using a three-step ABC method. The results showed tumor cell positively for SOX2 (Fig. 1E), CD34, p53, and AE1/AE3 and nefativity for desmin, S100P, SMARCA4 (Fig. 1C), and SMARCA2 (Fig. 1D).

Based on the results of the immunohistochemical analysis and histological features, we concluded that this tumor was a SMARCA4-DTS. In this case, the histological and immunohistochemical findings well representaed the characteristics of SMARCA4-DTS; if frozen material remains, genetic analysis may reveal SMARCA4 deficiencies or mutations.

An autopsy revealed a ganglioneuroma in the adrenal gland (Fig. 2A) and an enchondroma in the left clavicle (Fig. 2B). In other words, two of the three signs of Carney's triad were found; therefore, the patient was diagnosed with an incomplete form of carney's triad.

\section{Discussion And Conclusions}

SMARCA4-DTS is a group of diseases first reported by Le Loarer et al. in 2015 [1]. Their study investigated unclassified sarcoma by RNA sequencing and found that 19 cases showed inactivation of SMARCA4, which encodes the ATPase subunit of the BAF chromatin remodeling complex. SMARCA4- 
DTS has a poor prognosis, with a median survival of 7 months [7]. Therefore, the development of effective treatment is urgently needed. A SMARCA4 mutant lung cancer cell line was recently reported to show oxidative phosphorylation (oxphos) activity and sensitivity to its inhibitor IACS-010759 [11]. Thus, this drug may be an effective treatment for SMARCA4-DTS. In contrast, ARID1A was recently reported to promote the expression of SLC7A11 [12], which regulates the synthesis of antioxidant glutathione. Moreover, ARID1A-deficient cancers have lower glutathione levels owing to reduced SLC7A11 expression. Therefore, research is underway regarding the treatment of ARID1A-deficient cancer by inhibiting glutathione and its synthesis [12]. Therefore, since BRG1 is another factor responsible for the chromatin remodeling mechanism together with ARID1A, we hypothesized that SLC7A11 expression was decreased in SMARCA4-deficient tumors and ARID1A-dificient tumors. Subsequently, the expression levels of ARID1A and SLC11A were examined immunohistochemically. While a partial ARID1A deficiency was observed, SLC11A expression did not decrease. Thus, BRG1 deficiency was not directly related to SLC11A expression, and SLC11A expression requires the presence or absence of ARID1A deficiency.

A ganglioneuroma and enchondroma were found in the adrenal gland and left clavicle, respectively. Carney's triad is a rare multiple neoplastic association of pulmonary chondroma, gastrointestinal stromal tumor, and paraganglioma [9]. At least two tumors are required for its diagnosis [10]. Therefore, this case was considered an incomplete Carney's triad. Ganglioneuroma and chondroma may be less associated with SMARCA4-DTS but may be a subtype of the Carney complex.

In conclusion, we encountered a rare case of SMARCA4-DTS that showed typical histopathological and immunostaining findings. Further case reports nay help understand the pathobiological properties of SMARCA4-DTS and contribute to the general understanding of SMARCA4-DTS. More rarely, this case was accompanied by an incomplete form of Carney's triad consisting of chondroma and ganglioneuroma. This was a very rare in which two rare disease were combined.

\section{Abbreviations}

ARID1A: AT-rich interactive domain-containing protein 1A

BRG1: SMARCA4

oxphos: oxidative phosphorylation

S100P: S100 calcium-binding protein $\mathrm{P}$

SLC7A11: solute carrier family 7 member 11

SMARCA4: SWI/SNF-erlated, matrix-associated, actin-dependent regulator od chromatin, subfamily A, member 4

SMARCA4-DTS: SMARCA4-deficient thoracic sarcoma 
SOX2: sex-determining region Y-box 2

\section{Declarations}

Ethics approval and consent to participate: not applicable.

Consent for publication: not applicable.

Availability of data and materials: All data generated or analyzed during this study are included in this published article.

Consent: Written informed consent was obtained from the patient's family for ths publication of this case report and accompanying images.

Competing interest: The authors declare that they have no competing interests.

Funding: The authors declare that this paper received no financial support.

Authors' contributions: YK was major contributor in writing the manuscript. KK, CS, MH, SN, TM and YH participated in histological/gross evaluations. YM participated in clinical data collection. TT participated in the report conception and histological evaluation. All authors read and approved the final manuscript.

Acknowledgement: Not applicable.

Authors' information: Not applicable.

\section{References}

1. Francois L, Loarer sarah, Watson F, Tirode, et al. SMARCA4 inactivation defines a group of undifferentiated thoracic malignancies transcriptionally related to BAF-deficient sarcomas. Nat Genet. 2015;10:1200-5.

2. Tang L, Nogales E, Ciferri C. Structure and function of SWI/SNF chromatin remodeling complexes and mechanistic implications for transcription. Prog Biophys Mol Biol. 2010;102(2-3):122-8.

3. Zhang Y, Corey L, Smith C, Bustamante, et al. DNA translocation and loop formation mechanism of chromatin remodeling by SWI/SNF and RSC. Mol Cell. 2006;24(4):559-68.

4. jiang I Wu, Lessard J, Gerald R, Crabtree. Understanding the words of chromatin regulation. Cell. 2009;136(2):200-6.

5. Abbas Agaimy D, Jain N, Uddin, Lisa M, Rooper, JustinA Bishop. SMARCA4-deficient Sinonasal Cracinoma: A Series of 10 cases Expamding the Genetic Spectrum of SWI/SNF-driven Sinonasal Malignancied. Am J Surg Pathol. 2020;44(5):703-10.

6. Raul Perret L, Chalabreysse F le Loarer, et al. AMARCA4-deficient Thoracic Sarcomas: Clinicopathologic Study of 30 cases With an Emphasis on Their Nosology and Differential 
diagnoses. Am J Surg Pathol. 2019;43(4):455-465.

7. Jennifer L, Sauter RP, Graham, Jemmifer M, Boland, et al. SMARCA4-deficient thoracic sarcoma: adistinctive clinicopathological entity with undifferentiated rhabdoid morphology and aggressive behavior. Mod Pathol. 2017;10:1422-32.

8. Akihiko Toshida E, Konayashi N, Hitaoka, et al. Clinicopathological and molecular characterization of SMARCA4-deficient thoracic sarcomas with comparison to potentially related entities. Mod Pathol. 2017;6:797-809.

9. Caney JA, Sheps SG, Go VL, Gordon H. The triad of gastric leiomyosarcoma, functioning extraadrenal paraganglioma and pulmonary chondroma. N Engl J Med. 1977;296(26):1517-8.

10. Carney JA. Gastric stromal sarcoma, pulmonary chondroma, and extra-adrenal paraganglioma (Carney Triad): natural history, adrenocortical component, and possible familial occurrence. Mayo Clin Proc. 1999;74(6):543 - 52.

11. Deribe YL, Sun yuting, Futreal PA, et al. Mutations in the SWI/SNF complex induce a targetable dependence on oxidative phosphorylation in lung cancer. Mat Med. 2018;24(7):1047-57.

12. Hideaki Ogiwara K, Khno T, et al. Tageting the Vulneravility of Glutathione Metabolism in ARID1ADeficient Cancers. Cancer Cell. 2019;35(2):177-90.

\section{Figures}



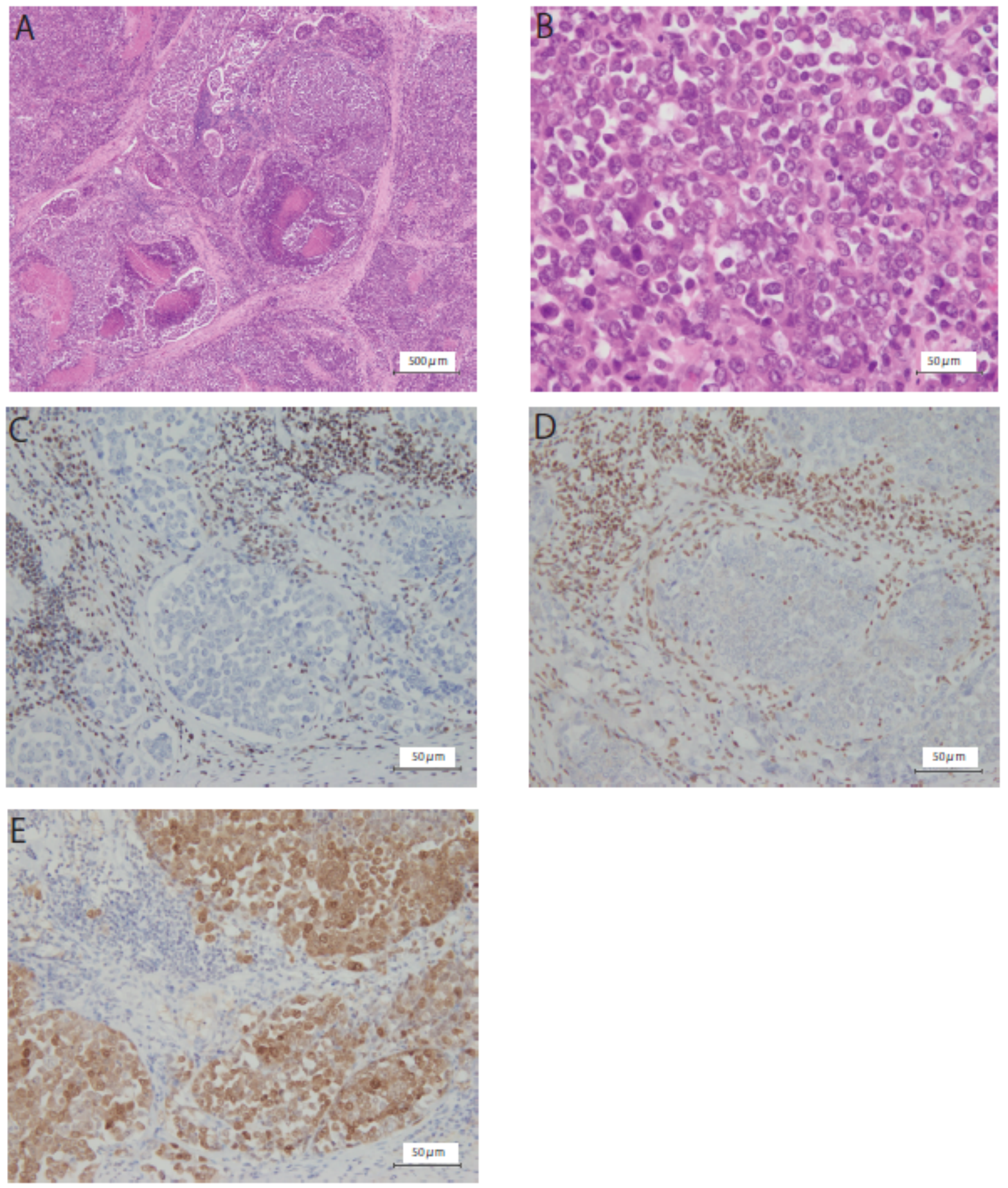

\section{Figure 1}

Representative histological findings and immunohistochemical features of SMARCA4-deficient thoracic sarcoma. A) Necrotic and sheet-like growth pattern of monotonous epithelial-like cells. B) The loosebinding tumor cells have distinct nucleoli; rhabdoid-like cells are also observed. C) Little or no immunoreactivity with anti-SMARCA4 antibody. D) Little or no immunoreactivity with ant-SMARCA2 antibody. E) Positive SOX2 immunoreactivity. 

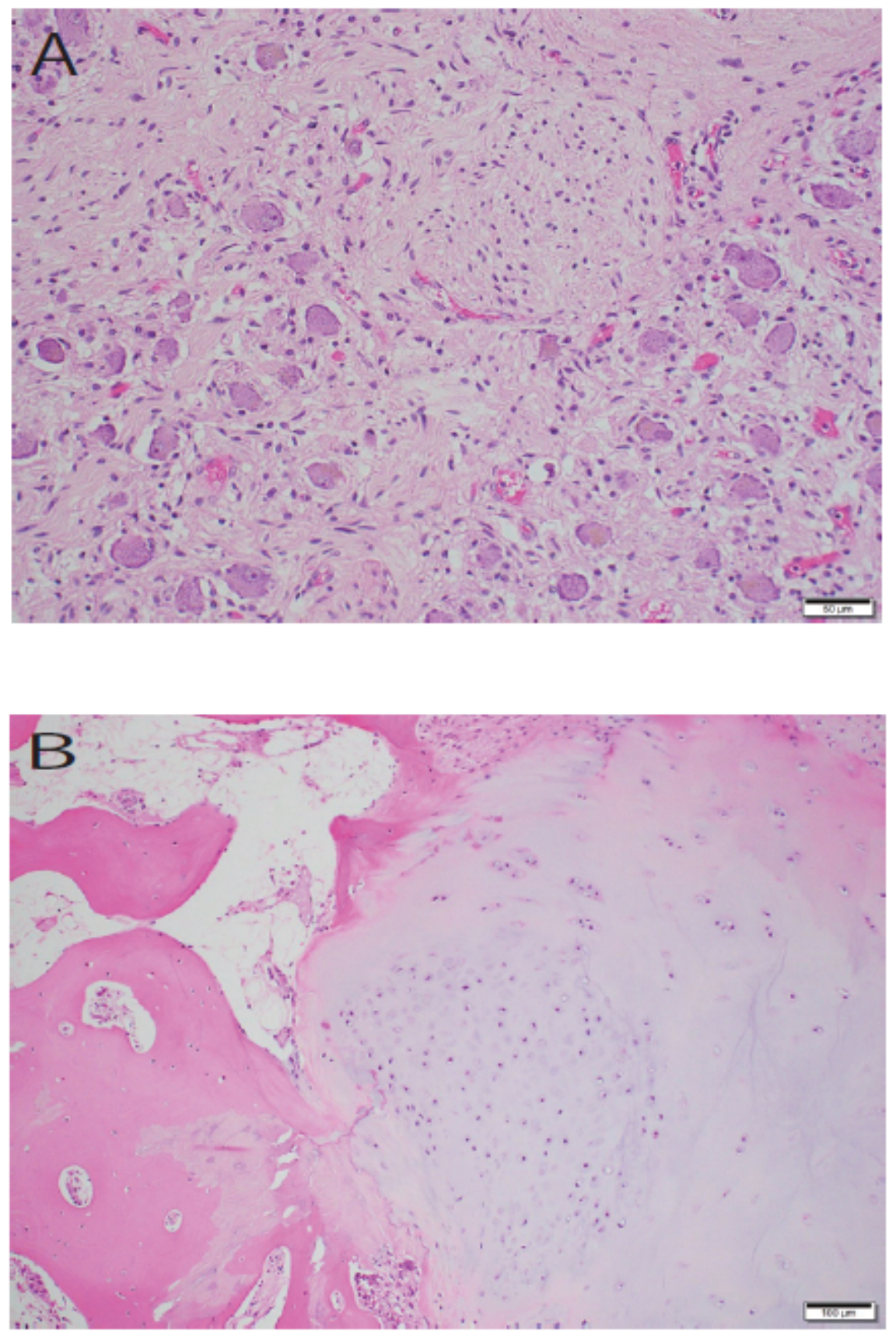

\section{Figure 2}

Repersentative histological findings of the ganglioneuroma in the adrenal gland $(A)$ and enchondroma in the left clavicle (B).

\section{Supplementary Files}

This is a list of supplementary files associated with this preprint. Click to download.

- CAREchecklistEnglish2013.pdf 